\title{
Light localization in azimuthally modulated Bessel photonic lattices
}

\author{
Robert Fischer - Dragomir N. Neshev $\cdot$ Servando López-Aguayo \\ Anton S. Desyatnikov · Andrey A. Sukhorukov • \\ Wieslaw Krolikowski · Yuri S. Kivshar
}

(C) Springer Science+Business Media, LLC 2007

\begin{abstract}
We study the propagation and localization of light beams in azimuthally modulated Bessel photonic lattices induced optically in photorefractive crystals; such lattices resemble highly nonlinear micro-structured fibres. We report on the experimental observation of discrete diffraction, nonlinear localization, and subsequent formation of spatial solitons. Our experimental results are confirmed by direct numerical simulations.
\end{abstract}

\section{Introduction}

When a high-intensity light beam propagates in a nonlinear self-focusing medium, it experiences self-trapping and spatial localization in the self-induced waveguide structure forming a spatial soliton. Solitons have been identified in a variety of nonlinear media [1]. They are highly robust objects exhibiting particle-like behaviour in interactions. This feature makes them promising candidates for beam control and all-optical switching. In recent decades, many research efforts have been devoted to the study of self-trapping of light and soliton formation in different types of media including, in particular, photonic structures with a periodic

R. Fischer · D. N. Neshev · S. López-Aguayo ·

A. S. Desyatnikov - A. A. Sukhorukov - W. Krolikowski ( $\square)$.

Y. S. Kivshar

Nonlinear Physics Centre and Laser Physics Centre, Centre for

Ultrahigh-bandwidth Devices for Optical Systems (CUDOS),

Research School of Physical Sciences and Engineering,

Australian National University, 0200 Canberra, ACT, Australia

e-mail:wzk111@rsphysse.anu.edu.au

S. López-Aguayo

Photonics and Mathematical Optics Group, Tecnológico de Monterrey, Monterrey, Mexico modulation of the refractive index (optical lattices). The spatially varying refractive index in nonlinear media affects the propagation and localization of optical beams. Periodicity results in the appearance of a photonic bandgap transmission spectrum for linear waves. This, in turn, modifies diffractional properties of waves reflected in their ability to experience either normal or anomalous diffraction. Latticeinduced propagation effects become even more dramatic in combination with the nonlinear response of the medium leading, for instance, to the formation of discrete and gap solitons [2]. Among a variety of nonlinear media photorefractive crystals gained a great attention because they deliver a strong nonlinearity at the microwatts laser power [3]. In addition, these crystals provide a convenient method to create periodic structures of various symmetries [4] which can be formed by an interference of several plane waves inside the crystal. The resulting stationary distribution of the light intensity leads to modulation of the crystal refractive index via a photorefractive effect. This technique is schematically illustrated in Fig. 1a, where four ordinary polarized plane waves overlap inside the crystal, which is biased externally with the DC electric field. As a result, a square lattice light intensity distribution induces the corresponding refractive index pattern, or a photonic lattice, in the crystal. This lattice can be then probed with another, extraordinarily polarized beam which, for sufficiently high intensity experiences self-focusing or defocusing leading to the formation of various types of solitons.

Instead of the holographic technique for generating optical lattices, we may employ nondiffracting beams with a transversely modulated intensity profile such as, for example, the Bessel-shaped beams. Many novel nonlinear effects have been predicted theoretically to occur in such ring-like optical lattices [5, 6]. The first experimental results on the formation of Bessel lattices and subsequent beam trapping 


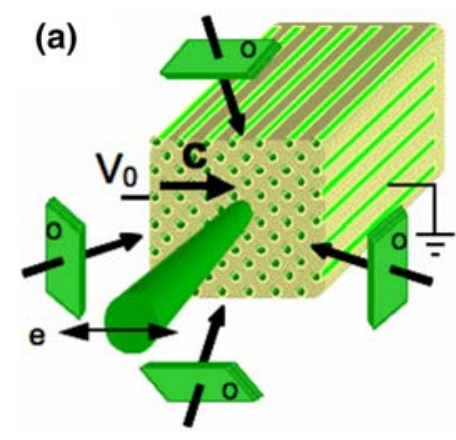

Fig. 1 Schematic illustration of the formation of optical lattice in a photorefractive crystal. (a) multi-wave interference; (b) use of the non-diffracting beam. In both cases labels "o" and "e" denote

have been reported very recently [7]. Due to their azimuthal symmetry, these ring-like lattices exhibit properties analogous to those of microstructured optical fibres. Hence they can be used to study both light localization and soliton formation in highly nonlinear multi-core photonic fibres.

Nondiffracting optical beams appear as a special class of linear modes propagating in the free space without any profile distortion [8]. The simplest example is a beam with the amplitude represented by the zero-order Bessel function and, therefore, such beams are commonly called Bessel beams. In this work, first we demonstrate the generation of different types of Bessel beams by a phase imprinting technique [9]. We then employ the nondiffracting nature of the azimuthally modulated Bessel beams to induce optical lattices in a biased photorefractive crystal, as shown schematically in Fig. 1b. These lattices are used to investigate nonlinear trapping of light beams. While nondiffracting beams are extensively employed for optical manipulation of small particles [10] and cold atoms [11], we show that such lattices can be used for the transverse optical trapping of localized light beams due to the selffocusing nonlinear response of the material [12].

\section{Generation of Bessel lattices}

A Bessel beam represents a particular solution of the wave equation such that any cross-section of the beam intensity distribution remains independent of the propagation variable $z$. Despite the fact that Bessel beams appear as eigensolutions of Maxwell's equation of infinite transverse extent and energy, they have been demonstrated in experiments with finite beams, where the characteristics of stationary propagation are sustained over long distances [13-16]. In past years, a number of practical ways to generate Bessel beams have been suggested including the use of ring apertures and amplitude masks [8], Fabry-Perot etalons, axicons, and holographic diffractive elements. Here, we employ a programmable phase modulator (PPM) (b)

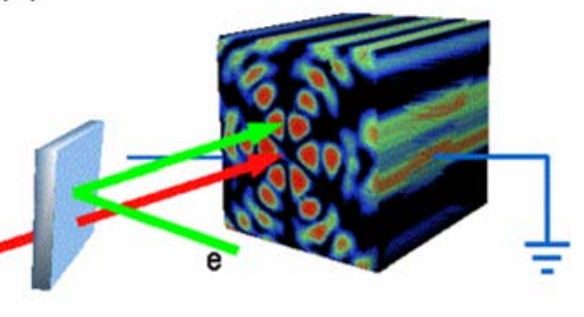

ordinary polarized lattice beams and extraordinary polarized probe beam, respectively; $\mathrm{V}_{0}$ - biasing DC voltage; vector $\vec{c}$ indicates the direction of optical axis of the crystal

and generate nondiffracting Bessel beams based on the phase-imprinting technique [9, 17]. We then study the propagation and self-action of probe beams in optical lattices induced by such Bessel beams.

In our experiments, a beam from a frequency-doubled cw Nd: $\mathrm{YVO}_{4}$ laser $(532 \mathrm{~nm})$ is split into two orthogonally polarized beams by a polarizing beam splitter (PBS), as shown in Fig. 2. The beam transmitted through the PBS is expanded approximately 10 times, and is sent to a phase modulator (Hamamatsu X8267). The modulator is programmed to reproduce the phase profile of the modulated Bessel beam:

$E_{\text {Bessel }}(r, \varphi)=A J_{n}(r / w) \cos (n \varphi)$,

where $r^{2}=x^{2}+y^{2}, \varphi=\tan ^{-1}(y / x)$ are the transverse polar coordinates, $w$ is the spatial scale, and $J_{n}$ is the $n$-th order Bessel function. Such a field distribution represents a well-known non-diffracting beam, and it is ideal for the application of the optical induction technique.

In practice, the input pattern distribution is superimposed onto a broad Gaussian carrier beam, hence the constant intensity profile is only preserved over a finite distance (focal region), which for our case of $w=7.6 \mu \mathrm{m}$ is approximately $20 \mathrm{~mm}$. In fact, the phase modulator only reproduces the phase structure of the Bessel beam and to obtain the correct amplitude modulation we employ Fourier filtering in the focal plane of the telescope, imaging the active plane of the modulator onto the front face of a $20 \mathrm{~mm}$ long SBN photorefractive crystal. The crystal is externally biased by a DC electric field $(3.5 \mathrm{kV} / \mathrm{cm})$ applied horizontally along the crystal c-axis, allowing the study of self-action effects.

\section{Light localization in a Bessel lattice}

The Bessel beam forming the lattice (Fig. 3a) is ordinary polarized and its stationary propagation inside the crystal is 


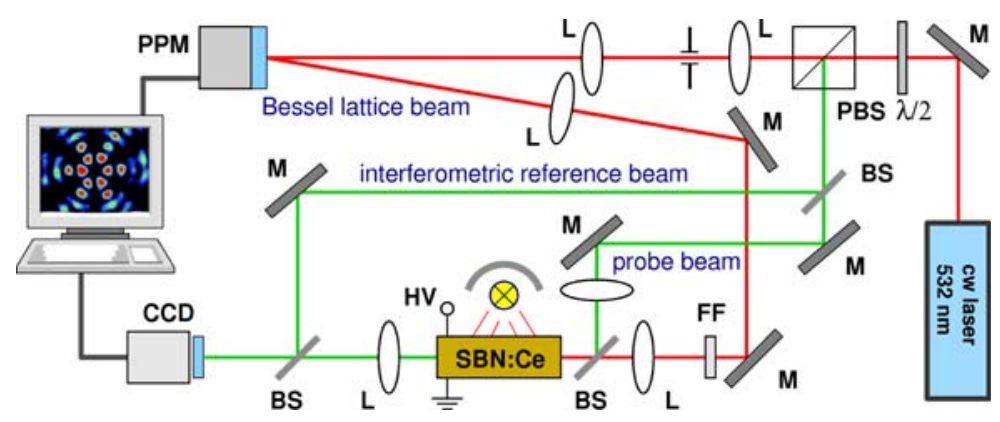

Fig. 2 Schematic structure of the experimental setup: PPM-programmable phase modulator; PBS-polarizing beam splitter; $\lambda /$ 2-half-wave plate; L-lenses; M-mirrors; BS—beam splitter;

not affected by the applied voltage due to the small value of the electro-optic coefficient for this polarization. However, this beam induces refractive index modulation in the crystal for the extraordinary polarized waves, and such a lattice reflects the Bessel-like spatial structure. Thus, an extraordinary polarized probe beam co-propagating in the crystal feels the induced Bessel-type index pattern and, simultaneously, experiences strong self-action due to the self-focusing photorefractive nonlinearity. As a probe we use the beam reflected from the PBS, which is combined with the lattice onto a beam splitter and is tightly focused to a size of $13.2 \mu \mathrm{m}$ onto the front face of the crystal. The input and output crystal faces can be imaged by a lens and recorded onto a CCD camera. Additionally, a small portion of the probe beam is used as an interferometric reference beam to monitor the phase profile of the probe beam at the output.

Without the lattice, the input probe beam (size shown as black circle in Fig. 3b) diffracts and increases its size to $162 \mu \mathrm{m}$ at the crystal output, as shown in Fig. $3 \mathrm{~b}(\sim 9$ diffractions lengths of propagation). After the PPM is switched on, a Bessel lattice following the intensity profile shown in Fig. 3a is induced in the crystal. It is important to note that we have to select the proper orientation of the lattice pattern with respect to the crystalline c-axis, in order to minimize the distortion of the refractive index modulation due to the anisotropy of the photorefractive crystal [18]. In our case of horizontal bias field, we find that the optimal orientation corresponds to a zero intensity line of the Bessel pattern perpendicular to the electric field, as shown in Fig. 3a. Still the anisotropy of the nonlinear response cannot be completely eliminated. The effect of anisotropy is different for the Bessel lattice of different orders. In our experiments, we have tested the azimuthally modulated Bessel lattices of the orders $n=1,2,3$, and 4 . For the low-order lattices $(n=1,2)$, the two or four sites in the inner ring dominate the induced refractive index structure, representing strong uncoupled waveguides. For
FF-Fourier filter; SBN: Ce—photorefractive crystal; CCD—camera. In red: Bessel lattice beam, in green: probe beam and interferometric reference beam

the higher-order Bessel beams $(n=4)$ (see Fig. 5) the effect of the crystal anisotropy leads to strong vertical merging (perpendicular to the c-axis) of the closely located lattice sites (Figs. 5c, d). Therefore, we first present the results for the representative case of the third-order modulated Bessel beams $(n=3)$.

When the probe beam propagates in the direction of the generated Bessel lattice, at low laser powers (20 nW) the beam experiences discrete diffraction with the output profiles strongly dependent on the specific location of the initial excitation. For excitation at the origin of the lattice the discrete diffraction is shown in Fig. 3c. At high power $(250 \mathrm{nW})$ the beam experiences nonlinear self-focusing and becomes localized at the six sites of the first ring of the Bessel lattice (Fig. 3d). For the input position between two upper sites of the main ring we observed beam localization at the power level of $600 \mathrm{nW}$ in the form of a double-peak, in-phase structure (Fig. 3f). For the same excitation geometry but at low powers $(20 \mathrm{nW})$ the beam diffracts as shown in Fig. 3e. Based on the generic properties of lattice solitons [1,2] and numerical simulations, we expect that the double-peak states are unstable with respect to symmetry breaking. When the input probe beam is positioned onto a lattice site of the first ring, the beam diffracts when its power is low (20 nW-Fig. 3g) and localizes to a single lattice site at a larger power (450 nW-Fig. $3 \mathrm{~h})$. In all the cases, the interferometric measurements show that the light localized on first lattice ring has constant phase (Fig. 4b). In Fig. 5 we illustrate linear and nonlinear propagation of light in optical lattice formed with the use of fourthorder Bessel beam. For central site excitation and input power of $450 \mathrm{nW}$ formation of localized state is evident (Fig. 5d). However, it is also clear that the lattice no longer possesses a circular symmetry. This is caused by strong anisotropy of the photorefractive effect, which causes the neighbouring maxima of the lattice to merge in vertical direction. 
Fig. 3 Experimental images:

(a) Intensity profile of the generated third-order Bessel lattice; (b) linear diffraction of the probe beam without lattice, the black circle shows the size of the beam at the crystal input; (c-h) linear diffraction (left) and nonlinear self-focusing (right) of the beam positioned $(\mathbf{c}, \mathbf{d})$ at the lattice centre, $(\mathbf{e}, \mathbf{f})$ between two cites, and $(\mathbf{g}, \mathbf{h})$ at a single site. The c-axis of the crystal and the biased field are horizontal
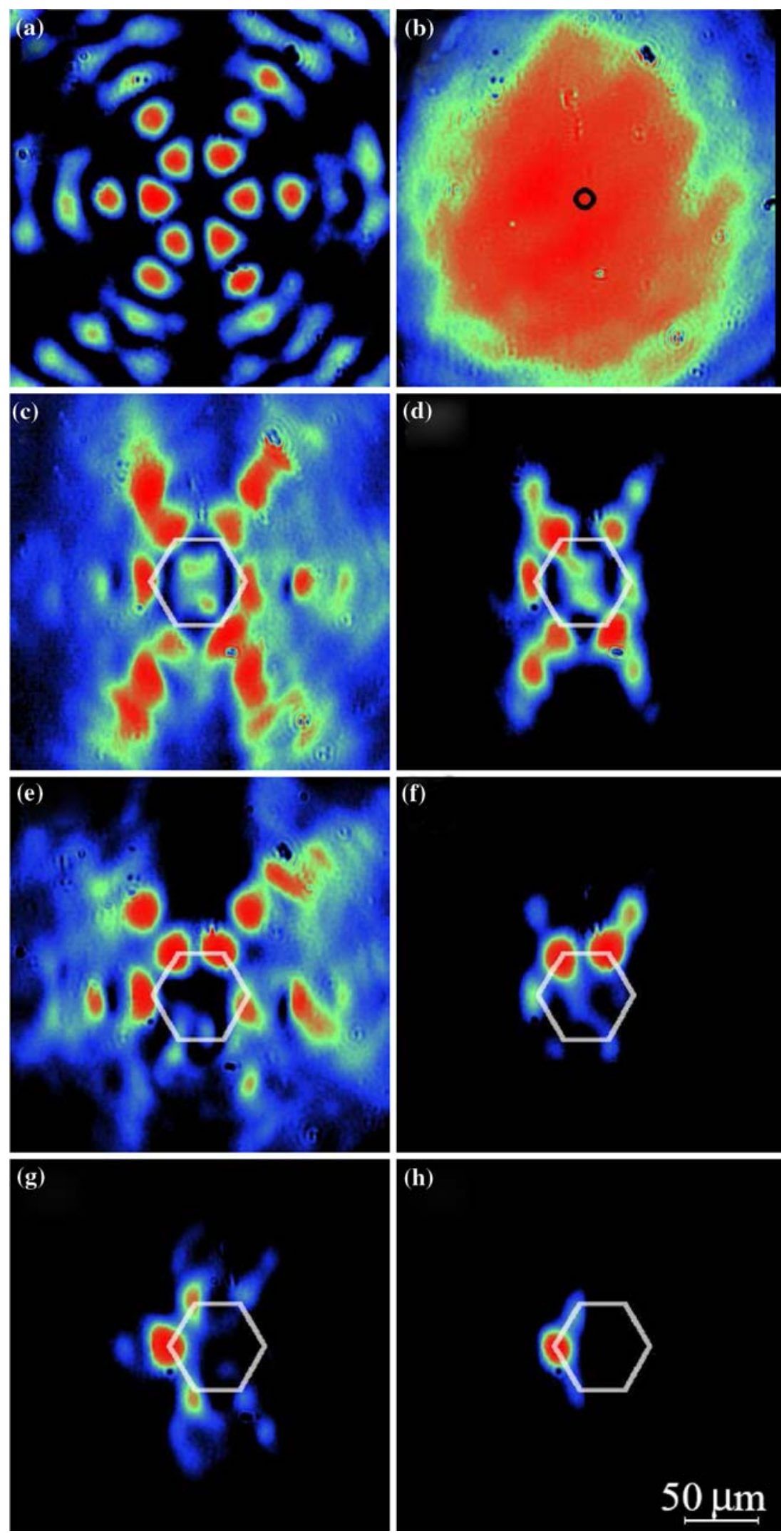
Fig. 4 (a) Experimentally observed intensity profile of the light localised on the first lattice ring; (b) corresponding interferogram indicating the same phase of the light maxima
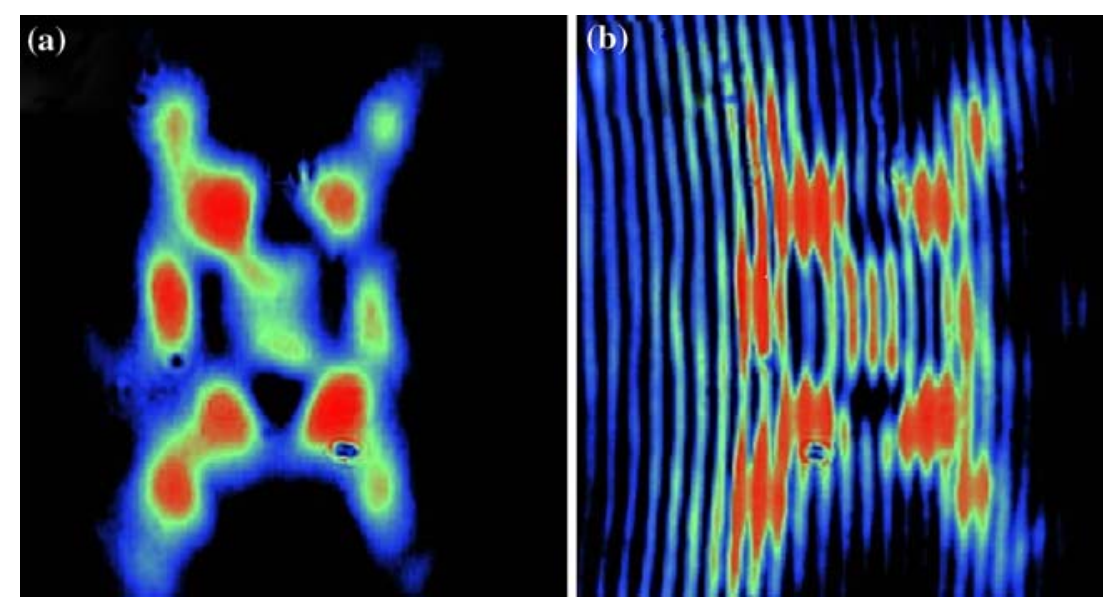

Fig. 5 Experimental results in the case of a fourth-order Bessel lattice $(n=4)$ : (a) intensity profile of the generated lattice; (b) linear diffraction of the probe beam without lattice, the black circle shows the size and position of the beam at the crystal input; (c) linear diffraction and (d) nonlinear self-focusing of the beam positioned at the lattice centre
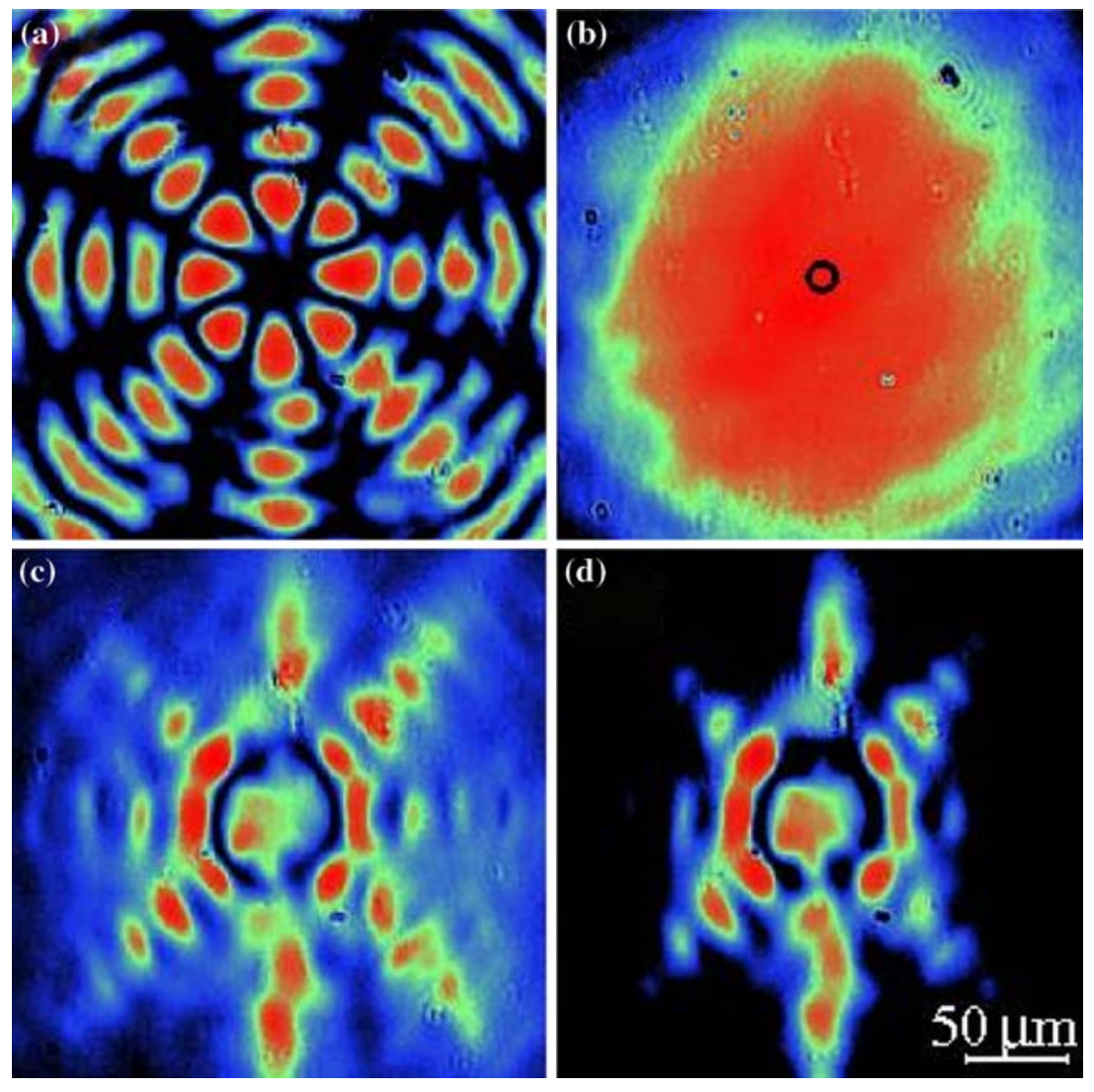

\section{Numerical results for the anisotropic model}

In order to confirm the existence of localized states corresponding to the experimental observations, we solve numerically the corresponding theoretical model accounting for the anisotropic and nonlocal nonlinear response of the photorefractive material [18]. This model describes the propagation of the beam with the electric field envelope $E(\mathrm{x}, \mathrm{y}, \mathrm{z})$, $i \partial_{z} E+\nabla^{2} E+\gamma \partial_{x} \varphi E=0$

coupled to the electrostatic potential $\varphi$ of the opticallyinduced space-charge field that satisfies the relation

$\nabla^{2} \varphi+\nabla \varphi \nabla \ln (1+I)=\partial_{x} \ln (1+I)$.

Here $\nabla^{2}=\partial_{x}^{2}+\partial_{y}^{2}$ and the physical variables $\tilde{x}, \tilde{y}$ and $\tilde{z}$ correspond to their dimensionless counterparts as 

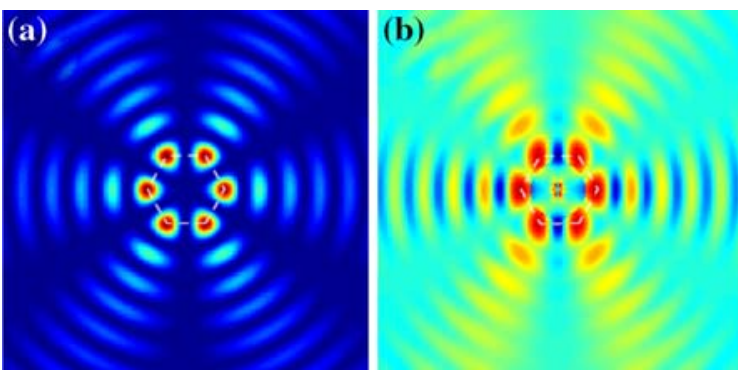

Fig. 6 Results of numerical simulations with the anisotropic nonlocal model Eqs. (3-5): (a) Intensity of the azimuthally modulated Bessel beam and (b) corresponding induced refractive index profile;

$(\tilde{x}, \tilde{y})=(w x, w y)$ and $\tilde{z}=2 k w^{2} z$ where $w$ is the transverse scale factor and $\kappa=2 \pi n_{0} / \lambda$ is the carrier wave vector with the linear refractive index $n_{0}$ and $\lambda$ is the wavelength. Parameter $\gamma=w^{2} \kappa^{2} n_{0}^{2} r_{\text {eff }} V$ determines the strength of nonlinearity and is defined through the effective electro-optic coefficient $r_{\text {eff }}$ and the externally applied bias DC field $V$. The total light intensity in the medium is given as

$I=|E|^{2}+A^{2} J_{3}^{2}(r) \cos ^{2}(3 \varphi)$,

where $I$ is measured in units of the background (dark) illumination intensity. We solve equation (3), (4) and find that the modulated Bessel beam induces a refractive index modulation, which has a symmetry lower than the symmetry of the intensity pattern (Figs. 6a, b). Stationary solutions of the system (2), (3) are sought in the standard form $E(x, y, z)=U(x, y) \exp (i \beta z)$, where the real envelope $U$ satisfies the equation

$-\beta U+\nabla_{\perp}^{2} U+\gamma \partial_{x} \varphi U=0$.

To find localized solutions in the system Eqs. (3-5) we apply the relaxation technique [17] with an initial profile in the form of a Gaussian ansatz. When the input beam is positioned in the origin of the optically-induced potential, the stationary solutions are ring-shaped (Fig. 6c), closely resembling the localized states observed in experiment (Fig. 3d). However, if the propagation constant $\beta$ exceeds some threshold, which corresponds to the threshold for soliton power and peak intensity, our relaxation procedure always converges to the on-site single soliton, shown in Fig. 6d. This indicates that two families of solutions may be linked through a bifurcation, which may correspond to the onset of symmetry-breaking instability for the effectively two-lobe "in-phase" soliton shown in Fig. 3f. However, this aspect requires further investigations.
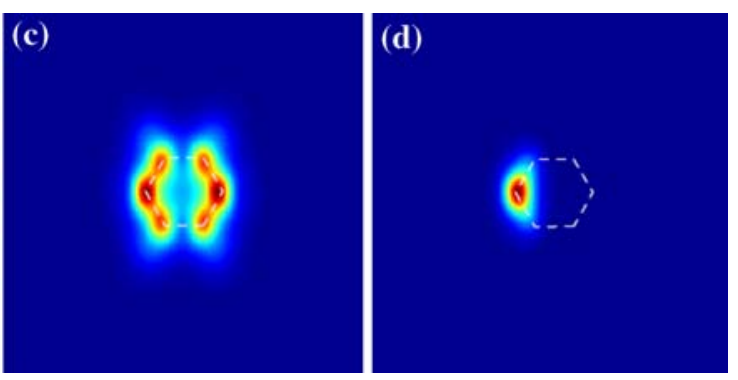

parameters are $A=2.5, \gamma=2$. (c, d) Examples of the ring-like and single-site solitons corresponding to the experimental data shown in Figs. 3d, h, respectively

\section{Conclusions}

We have generated experimentally the azimuthally modulated Bessel-like optical lattices in a photorefractive crystal by employing a phase-imprinting technique. We have found that nonlinear beam propagation in these segmented Bessel lattices is accompanied by light localization in the form of multi-site ring-shaped and single-site self-trapped states. Our experimental results are in a good agreement with numerical simulations based on an anisotropic model of photorefractive nonlinearity. Due to their azimuthal symmetry, the Bessel lattices can be considered as an analogue of multi-core highly nonlinear microstructured fibres, so our results can indicate possibility of light localisation in such fibers [19].

Acknowledgement The authors acknowledge a support from the Australian Research Council.

\section{References}

1. Yu.S. Kivshar, G.P. Agrawal, Optical Solitons: From Fibers to Photonic Crystals (Academic Press, San Diego, 2003)

2. D.N. Christodoulides, F. Lederer, Y. Silberberg, Nature 424, 817 (2003)

3. W. Krolikowski, B. Luther-Davies, C. Denz, IEEE J. Quant. Electron. 39, 3 (2003)

4. N.K. Efremidis, S. Sears, D.N. Christodoulides, J.W. Fleischer, M. Segev, Phys. Rev. E 66, 046602 (2002); D. Neshev, A. Sukhorukov, Y. Kivshar, E. Ostrovskaya, W. Krolikowski, Opt. Lett. 28, 710 (2003)

5. Y.V. Kartashov, V.A. Vysloukh, L. Torner, Phys. Rev. Lett. 93, 093904-4 (2004)

6. Y.V. Kartashov A.A, Egorov V.A. Vysloukh L. Torner, Phys. Rev. E 70, 065602-4 (2004)

7. X. Wang, Z. Chen, P.G. Kevrekidis, Phys. Rev. Lett. 96, 0839044 (2005)

8. J. Durnin, J. Opt. Soc. Am. A 4, 651 (1987)

9. N. Chattrapiban, E.A. Rogers, D. Cofield, W.T. Hill, R. Roy, Opt. Lett. 28, 2183 (2003)

10. D. McGloin, K. Dholakia, Contemp. Phys. 46, 15 (2005) 
11. D. McGloin, G.C. Spalding, H. Melville, W. Sibbett, K. Dholakia, Opt. Expr. 11, 158 (2003)

12. R. Fischer, D. Neshev, S. Loppez-Aguayo, A. Desyatnikov, A. Sukhorukov, W. Krolikowski, Y. Kivshar, Opt. Express 14, 2825 (2006)

13. J. Durnin, J.J. Miceli Jr., J.H. Eberly, Phys. Rev. Lett. 58, 1499 (1987)

14. Y. Lin, W. Seka, J.H. Eberly, H. Huang, D.L. Brown, Appl. Opt. 31, 2708 (1992)

15. T. Wulle, S. Herminghaus, Phys. Rev. Lett. 70, 1401 (1993); Erratum: Phys. Rev. Lett. 71, 209 (1993)
16. C. Lopez-Mariscal, J.C. Gutierrez-Vega, S. Chavez-Cerda, Appl. Opt. 43, 5060 (2004)

17. S.H. Tao, W.M. Lee, X.C. Yuan, Opt. Lett. 28, 1867 (2003)

18. A.A. Zozulya, D.Z. Anderson, A.V. Mamaev, M. Saffman, Phys. Rev. A 57, 522 (1998)

19. A.B. Fedotov, P. Zhou, A.N. Naumov, V.V. Temnov, V.I. Beloglazov, N.B. Skibina, L.A. Melnikov, A.V. Shcherbakov, A.P. Tarasevitch, D. von der Linde, A.M. Zheltikov, Appl. Phys. B 75, 621 (2002) 